\title{
Different clinical symptom patterns in patients with reflux micro-aspiration
}

\author{
Oleksandr Khoma $\mathbb{C}^{1,2}$, Jin-soo Park ${ }^{1,2}$, Felix Michael Lee ${ }^{2}$, Hans Van der Wall ${ }^{1,3}$ and Gregory L. Falk ${ }^{2,4,5}$
}

${ }^{1}$ University of Notre Dame, Freemantle, Australia. ${ }^{2}$ Dept of Upper Gastro-Intestinal Surgery, Concord Repatriation General Hospital, Sydney, Australia. ${ }^{3}$ Concord Nuclear Imaging, Sydney, Australia. ${ }^{4}$ Sydney Heartburn Clinic, Lindfield, Australia. ${ }^{5}$ University of Sydney, Sydney, Australia.

Corresponding author: Oleksandr Khoma (khoma.a@gmail.com)

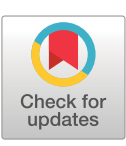

This version is distributed unde the terms of the Creative Commons Attribution NonCommercial Licence 4.0. For commercial reproduction rights and permissions contact permissions@ersnet.org

Received: 17 Aug 2021 Accepted: 26 Oct 2021

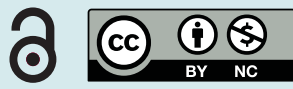

Shareable abstract (@ERSpublications)

Patients with reflux micro-aspiration most commonly present with a combination of regurgitation and/or heartburn and cough and/or throat clearing https://bit.ly/3GM8cNS

Cite this article as: Khoma O, Park J-soo, Lee FM, et al. Different clinical symptom patterns in patients with reflux micro-aspiration. ERJ Open Res 2022; 8: 00508-2021 [DOI: 10.1183/ 23120541.00508-2021].

\section{Abstract}

Background Pulmonary manifestation of gastro-oesophageal reflux disease (GORD) is a well-recognised entity; however, little primary reported data exists on presenting symptoms of patients in whom reflux micro-aspiration is confirmed. The aim of this study is to report symptoms and presenting patterns of a large group of patients with confirmed reflux micro-aspiration.

Patients and methods Data was extracted from a prospectively populated database of patients referred to a tertiary specialist centre with severe, refractory or atypical reflux. Patients with reflux micro-aspiration on scintigraphy were included in this study. A separate group included patients with evidence of proximal reflux to the level of pharynx when supine and/or upright.

Results Inclusion criteria were met by 243 patients with confirmed reflux micro-aspiration (33\% males; mean age 59). Most common symptoms amongst patients with micro-aspiration were regurgitation (72\%), cough (67\%), heartburn (66\%), throat clearing (65\%) and dysphonia (53\%). The most common twosymptom combinations were heartburn/regurgitation, cough/throat clearing, regurgitation/throat clearing, cough/regurgitation and dysphonia/throat clearing. The most common three-symptom combinations were cough/heartburn/regurgitation, cough/regurgitation/throat clearing and dysphonia/regurgitation/throat clearing. Cluster analysis demonstrated two main symptom groupings, one suggestive of proximal volume reflux symptoms and the other with motility/inflammatory bowel syndrome-like symptoms (bloat, constipation).

Conclusion The combination of typical symptoms of GORD such as heartburn or regurgitation and a respiratory or upper aero-digestive complaint such as cough, throat clearing or voice change should prompt consideration of reflux micro-aspiration.

\section{Introduction}

Reflux micro-aspiration is a severe consequence of gastro-oesophageal reflux disease (GORD) [1] Although a well-recognised entity, understanding of the physiology, natural history or common presentation of patients with reflux micro-aspiration remains limited. Links between typical GORD symptoms such as heartburn and regurgitation and atypical symptoms (e.g. cough, globus pharyngeus) have been made $[2,3]$. Previously published data shows distinct presenting symptomatology amongst patients with reflux cough [4].

Correlation between reflux micro-aspiration and exacerbation of bronchial disease has been previously shown in small cohort studies of patients undergoing bronchoscopy [5, 6].

Multiple aero-digestive questionnaires are currently used, such as Reflux Symptom Index (RSI), Supraoesophageal Reflux Questionnaire (SERQ) and Hull Airway Reflux Questionnaire (HARQ), none of 
which have been validated in patients with proven reflux micro-aspiration largely due to lack of an adequate diagnostic test [1, 7-9].

No large-scale studies examining symptoms of patients with proven reflux micro-aspiration have been published so far.

The aim of this study is to analyse patterns of presenting symptoms in a large group of patients with proven pulmonary micro-aspiration by new technology reflux micro-aspiration scintigraphy (RASP). The secondary goal of this study was to analyse symptom patterns in patients with RASP for evidence of pharyngeal reflux whilst supine and upright.

\section{Patients and methods}

Data were extracted from the prospectively maintained database containing records of patients presenting with severe typical or atypical, treatment-resistant GORD.

All patients' clinical symptoms were recorded by a senior consultant at the time of the initial assessment using a standardised symptom sheet. Symptoms were recorded as either present or not. Severity of a specific symptom was not graded.

Patients that had evidence of pulmonary micro-aspiration of gastric refluxate on RASP performed between December 2006 and March 2018 were included in this study. We have also included patients with RASP evidence of proximal reflux to the level of pharynx when supine and/or upright were compared as a separate group which excluded patients with reflux micro-aspiration. There was an overlap amongst patients with proximal reflux supine and upright.

Scintigraphy technique included placing fasted overnight patients before a Hawkeye 4 gamma camera (General Electric, Milwaukee, WI, USA) with stomach, chest and upper airway in the field of view. Patients were administered 40-60 MBq of 99mTc DTPA diluted in $150 \mathrm{~mL}$ of water, followed by $50 \mathrm{~mL}$ of water to promote clearance of isotope from pharynx and oesophagus. Images were obtained for $2 \mathrm{~min}$ at 15 s per frame into a $64 \times 64$ matrix, followed by a 30 -min dynamic image while supine for $30 \mathrm{~s}$ per frame. Delayed images were obtained at $2 \mathrm{~h}$ to assess for presence of isotope in the lungs as evidence of reflux aspiration. Isotope time activity curves captured for the pharynx and upper oesophagus supine and erect were classified as falling, flat or rising curves, where a falling curve reflected clearance of refluxate and a rising curve accumulation of refluxate. Rising pharyngeal curves have been shown to be strongly associated with reflux aspiration [10]. Detailed description of the new modified technique of RASP, as well as validation study, have been previously published [10,11]. It is important to note that this not a standard scintigraphy, but a new, highly specific procedure with improved accuracy and has been instrumental in identifying previously silent micro-aspiration.

Symptoms were analysed for the most common symptom, as well as most frequent two- and three-symptom combinations in groups of patients with micro-aspiration, pharyngeal contamination supine and pharyngeal contamination upright. Results have been reported in absolute numbers and prevalence.

Cluster analysis of the principal variables (symptoms, micro-aspiration, pharyngeal contamination) was performed using Euclidean distance (root mean squared) and displayed as a vertical icicle plot.

The database was approved by the University of Notre Dame Australia Human Research Ethics Committee (019091S) on July 23, 2019, being previously approved by the Concord Hospital Ethics Committee (LNR/ $12 \mathrm{CRGH} / 248$ ). This study protocol conforms to the ethical guidelines of the 1975 Declaration of Helsinki. All patients provided informed written consent to participate in research.

\section{Results}

Inclusion criteria were met by 243 patients with reflux micro-aspiration. A further 230 patients with pharyngeal contamination when upright and 298 patients with pharyngeal contamination whilst supine were also included. Demographic data are summarised in table 1.

Rate of reflux micro-aspiration detection following referral for RASP was 26\% (946 referred, 243 confirmed positive for micro-aspiration). 


\section{TABLE 1 Demographic characteristics of the participants}

\begin{tabular}{|c|c|c|c|}
\hline & Micro-aspiration & $\begin{array}{l}\text { Pharyngeal contamination } \\
\text { supine }\end{array}$ & $\begin{array}{c}\text { Pharyngeal contamination } \\
\text { upright }\end{array}$ \\
\hline Subjects $\mathrm{n}$ & 243 & 230 & 298 \\
\hline Sex, female \% & 67 & 69 & 61 \\
\hline Age years, median (range) & $62(17-88)$ & $61(14-88)$ & $59(16-87)$ \\
\hline BMI $\mathrm{kg} \cdot \mathrm{m}^{-2}$, mean $\pm \mathrm{sD}$ & $27.1 \pm 3.93$ & $27.9 \pm 4.01$ & $27.3 \pm 2.82$ \\
\hline PPI use $\%^{\#}$ & 68 & 66 & 66 \\
\hline Prokinetic or laxative use $\%$ & 5 & 4 & 4 \\
\hline
\end{tabular}

\section{Reflux micro-aspiration}

The most common symptom in patients with reflux micro-aspiration was regurgitation (72\%), followed by cough (67\%) and heartburn (66\%). The most common two-symptom combination was heartburn and regurgitation, followed by cough and throat clearing. The most common three-symptom combination was cough, heartburn and regurgitation followed by cough, regurgitation and throat clearing. A summary of symptoms of patients with reflux micro-aspiration can be found in table 2 .

Patients self-reported medical diagnosis as "symptoms”, including asthma as a child (4.53\%), as an adult (5.35\%), pneumonia (5.76\%) and bronchitis (18.52\%). It was not possible to verify the accuracy of these diagnoses as well as the criteria on which they were based.

\section{Pharyngeal reflux contamination}

Patients with pharyngeal contamination most commonly presented with regurgitation (71\%), heartburn (68\%) and throat clearing (67\%). The most common two-symptom combination was heartburn/ regurgitation, followed by regurgitation/throat clearing and cough/throat clearing. The top three-symptom combination included heartburn/regurgitation/throat clearing, cough/regurgitation/throat clearing and dysphonia/regurgitation/throat clearing.

A summary of symptoms of patients with pharyngeal contamination supine and upright is presented in table 3.

\section{Cluster analysis}

Whilst no specific symptom clustering was observed in relationship to aspiration, two distinct symptom clusters emerged as demonstrated in figures 1 and 2. The first cluster was of laryngospasm, voice change,

\begin{tabular}{lc} 
TABLE 2 Symptom prevalence (\%) in patients with reflux micro-aspiration ( $\mathrm{n}=243)$ & 72.43 \\
Regurgitation & 67.49 \\
Cough & 66.67 \\
Heartburn & 66.26 \\
Throat clearing & 53.09 \\
Dysphonia & 43.62 \\
\hline Dyspnoea all & 42.39 \\
Sore throat & 25.10 \\
\hline Regurgitation (throat) & 23.87 \\
Laryngospasm & 22.63 \\
Chest pain atypical & 21.81 \\
Sleep disturbance & 20.99 \\
Dysphagia (slow) & 18.93 \\
Dyspnoea (exercise induced) & 17.70 \\
Bloating & 5.35 \\
Dyspnoea (post prandial) & 4.94 \\
Flatus & 3.70 \\
Voice change & 3.29 \\
\hline Regurgitation (low) & 1.23 \\
\hline Odynophagia & \\
\hline
\end{tabular}


TABLE 3 Symptom prevalence (\%) amongst patients with pharyngeal contamination whilst supine and upright

\begin{tabular}{lcc} 
Symptom & $\begin{array}{c}\text { Patients with supine pharyngeal } \\
\text { contamination }^{\#}\end{array}$ & $\begin{array}{c}\text { Patients with upright pharyngeal } \\
\text { contamination }^{*}\end{array}$ \\
\hline Regurgitation & 71.81 & 70.87 \\
Heartburn & 68.46 & 67.39 \\
\hline Throat clearing & 67.79 & 66.09 \\
Cough & 62.75 & 64.35 \\
Dysphonia & 57.05 & 54.35 \\
Sore throat & 47.32 & 46.09 \\
Mucus & 41.61 & 44.78 \\
Dyspnoea all & 38.26 & 42.17 \\
Globus sensation & 36.24 & 36.96 \\
Dysphagia (typical) & 28.52 & 28.70 \\
Laryngospasm & 23.15 & 28.26 \\
Bloating & 22.82 & 26.96 \\
Chest pain atypical & 22.82 & 25.22 \\
Regurgitation (throat) & 22.15 & 23.04 \\
Sleep disturbance & 21.81 & 22.17 \\
Dysphagia (slow) & 18.79 & 21.74 \\
Dyspnoea (exercise induced) & 14.09 & 16.52 \\
Early satiety & 10.07 & 13.48 \\
\hline Flatus & 6.71 & 6.52 \\
Voice change & 4.70 & 5.22 \\
\hline Dyspnoea (post prandial) & 3.69 & 4.35 \\
\hline Regurgitation (low) & 3.69 & 3.91 \\
\hline Odynophagia & 2.68 & 3.91 \\
\hline
\end{tabular}

\#: $n=230 ;{ }^{\bullet}:$ n=298.

Tree diagram for 12 variables

Single linkage

Euclidean distances

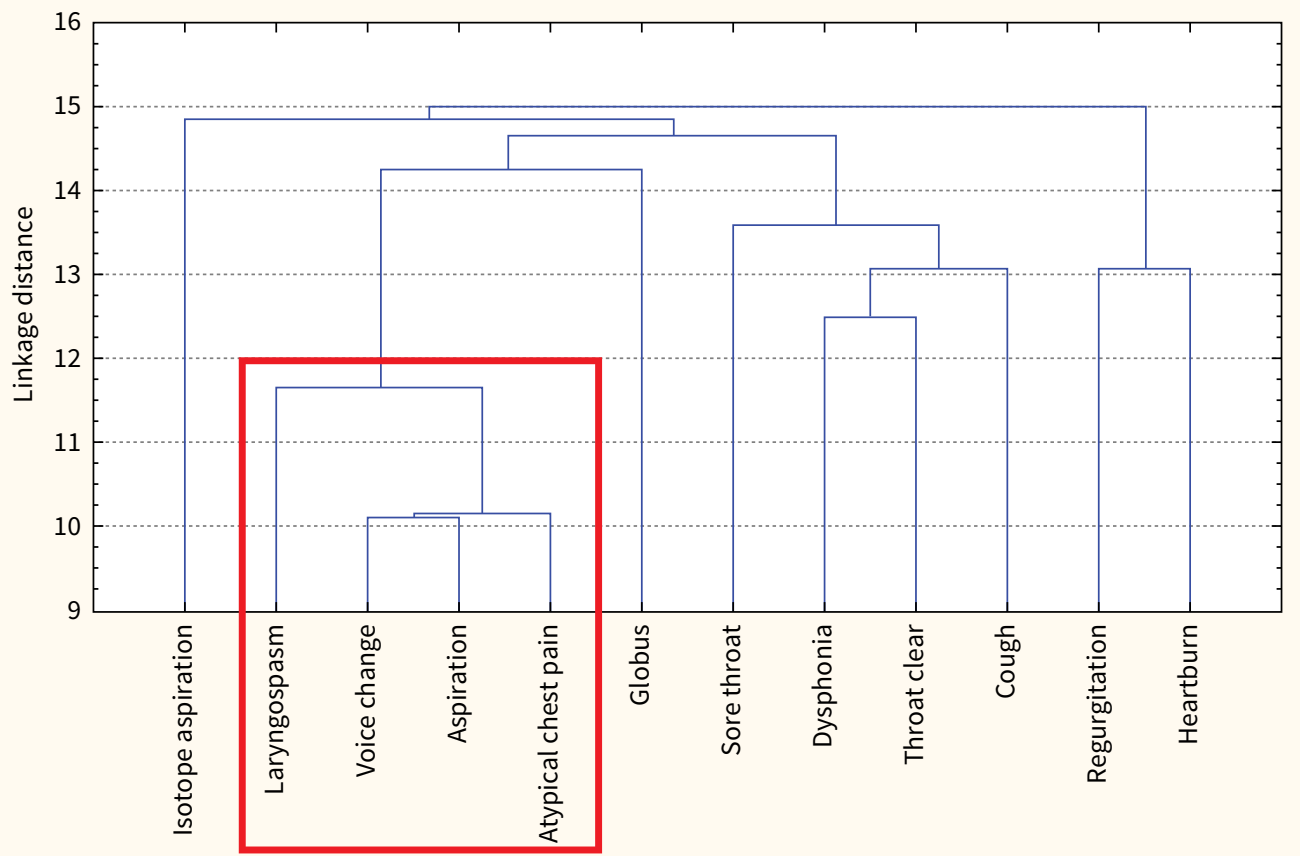

FIGURE 1 Cluster analysis of the principal variables demonstrating clustering of symptoms of laryngospasm, voice change, aspiration and atypical chest pain. 


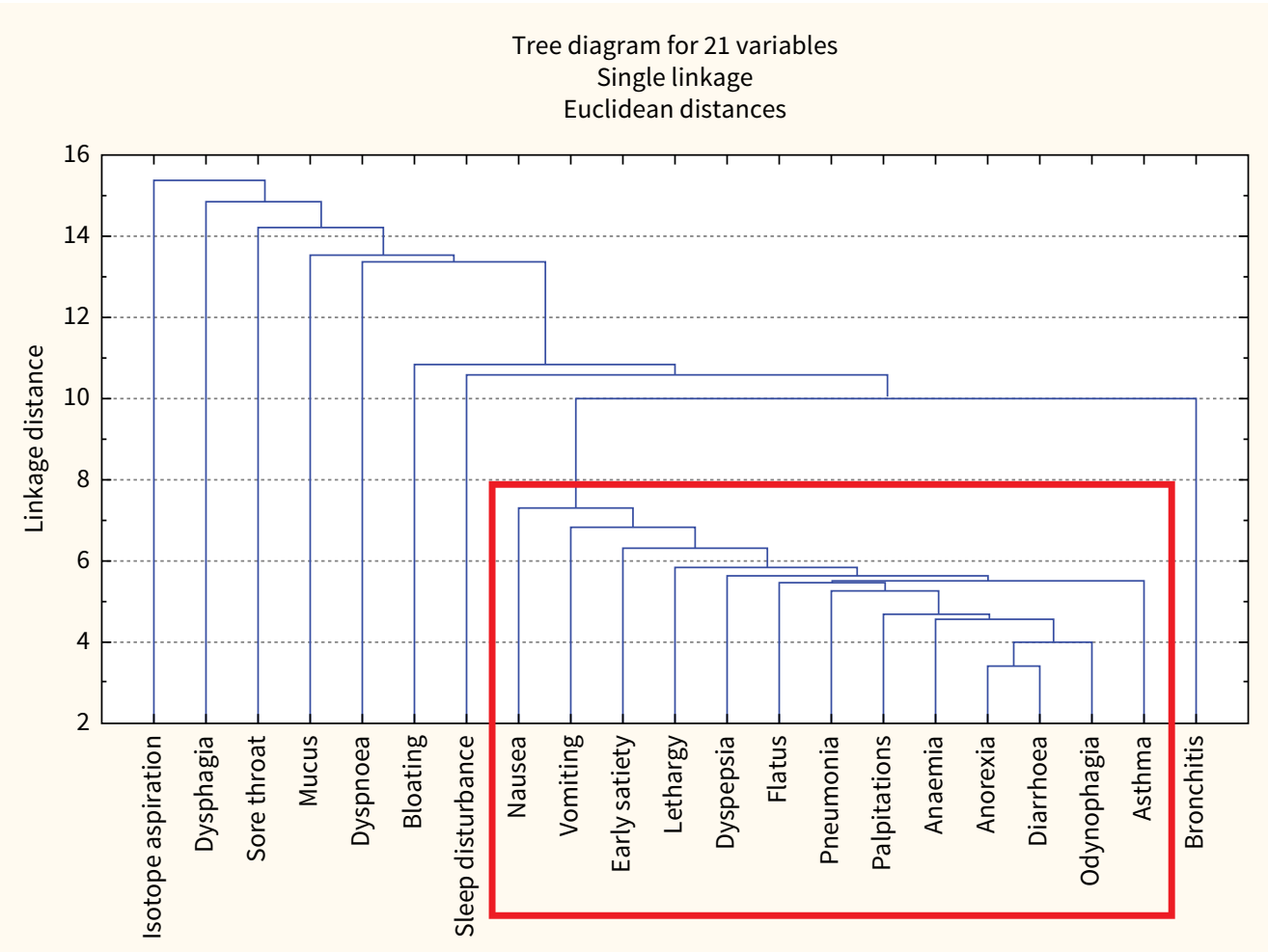

FIGURE 2 Cluster analysis of the principal variables demonstrating clustering of symptoms of nausea, vomiting, early satiety, bloating, flatus, pneumonia, asthma, diarrhoea, dyspepsia and lethargy.

aspiration and atypical chest pain and second cluster was of nausea, vomiting, early satiety, bloating, flatus, pneumonia, asthma, diarrhoea, dyspepsia and lethargy.

Discussion

Aspiration of gastric refluxate has been long recognised as a less common form of GORD [12-15]. It can lead to pulmonary complications including adult-onset asthma, chronic cough and pulmonary fibrosis [16-20].

Reflux micro-aspiration has been suspected in adults and children with chronic unexplained respiratory symptoms. However, no current recommendations exist in whom possibility of GORD should be interrogated early for fear of pulmonary disease [21-25].

The diagnosis of atypical or extra-oesophageal manifestations of GORD can be challenging. For example, patients presenting with laryngo-pharyngeal reflux (LRP) to this service tend to be 8 years older than those presenting with typical GORD, many of whom spent years between subspeciality medical services without diagnosis [10].

The aim of this study was to examine common clinical symptom patterns amongst patients with proven reflux aspiration. We have discovered that commonly patients with reflux aspiration would present with a combination of typical GORD symptoms (most commonly regurgitation and heartburn) and respiratory symptoms such as cough or throat clearing.

Cluster analysis revealed two distinct patterns of symptoms clustering. The first cluster included aspiration associated with laryngospasm, voice change and atypical chest pain, most suggestive of volume proximal reflux and possibly impaired pharyngeal guarding and/or clearance. The second cluster included symptoms of nausea, vomiting, early satiety, bloating, flatus, pneumonia, asthma, diarrhoea, dyspepsia and lethargy suggestive of motility-related irritable bowel syndrome-like symptoms which were associated with pulmonary complications of the reflux aspiration. This raises the question of reflux aspiration being associated with IBS in a group of patients. Global gut dysmotility has been proposed as one of the pathophysiological explanations for IBS [26]. Furthermore, bloating and constipation has been associated 
with refractory GORD and laryngopharyngeal reflux (LPR) [27]. Can two of the diseases (IBS and reflux aspiration) be related? We have previously demonstrated a link between dysmotility and aspiration [10] and in this study have demonstrated clustering of IBS symptoms in patients with proven reflux aspiration. The connection seems plausible; however, specific studies in patients with confirmed IBS are needed to test for a possible relationship.

Typical GORD symptoms such as regurgitation can be overlooked by clinicians and not presented by patients as both concentrate on heartburn. The results of this study demonstrated this to be the most common symptom in patients with reflux aspiration and made apparent by experienced questioning. We therefore suggest taking focused GORD symptom history in patients presenting with chronic cough unexplained by most common causes such as smoking, angiotensin-converting enzyme (ACE) inhibitor use and post-nasal drip. The use of validated questionnaires such as Hull Airways Reflux Questionnaire can be helpful to further select patients requiring a confirmatory test to demonstrate aspiration or proximal pharyngeal reflux.

Patients with reflux micro-aspiration can present with isolated respiratory complaints, usually cough, with little-to-no typical symptoms of GORD such as heartburn or retrosternal chest pain. Multiple hypotheses have been proposed to explain this including aerosolised refluxate (airway reflux) producing little irritation to the digestive system [12]. Amongst these patients eliciting symptoms related to the oesophageal dysmotility (such as slow transit dysphagia, globus, bloating and early satiety) can increase the index of suspicion of airway reflux as ineffective oesophageal motility is strongly associated with reflux micro-aspiration [10].

Proton pump inhibitors (PPIs), which were prescribed in close to $70 \%$ of patients in this study, can mask some of the typical GORD symptoms by reducing the acidity of the refluxate. Whilst reducing the acidity of the refluxate, PPIs have not been shown to reduce the rate of reflux events, and possibly increase aspiration risk as the rates of community-acquired pneumonia are increased amongst PPI users [28, 29].

Conventional GORD investigations such as endoscopy, impedance and $\mathrm{pH}$ monitoring have limited sensitivity in detecting proximal reflux and are unable to demonstrate reflux aspiration. Use of invasive tests such as salivary or bronchial lavage testing for pepsin has had conflicting evidence [5, 6, 30]. Patients with suspected reflux aspiration should therefore proceed on to confirmatory tests such as the modern precise reflux scintigraphy, which is inexpensive and noninvasive with excellent specificity [1, 11].

Multiple hypotheses of causative relationships between cough and reflux have been proposed including oesophago-bronchial reflex hypersensitivity, laryngeal irritation by LPR and micro-aspiration [9, 31]. The results of our study where participants were confirmed to have pulmonary reflux micro-aspiration and proximal (LPR) reflux would support both above-mentioned hypotheses.

A limitation of this study was in significant pre-selection bias as the decision to refer patients for RASP was based on the clinical judgement of a senior treating clinician. Generally, patients with a combination of typical GORD symptoms and presence of extra-oesophageal symptoms or discordant findings of clinical history and impedance or manometry would prompt RASP study. Since RASP is not used to investigate all patients referred to this service, selection bias was introduced when patients were referred for scintigraphy. It was therefore difficult to perform comparison with patients in whom RASP study was negative for micro-aspiration, as the pre-test probability was very high by the virtue of referral to a tertiary reflux institution and further pre-test selection by clinical suspicion based on history. Therefore, results cannot be generalised to all patients with typical or atypical GORD. A further limitation was lack of a control group to assess the specificity of the symptom patterns. Another limitation of this study is a lack of use of validated questionnaires limiting ability to assess the sensitivity of such questionnaires.

Possible bias may have been introduced by modification of the RASP technique, most significantly by addition of SPECT/CT in 2017. This is thought to increase sensitivity and specificity of the study; however, to date this has not been shown to increase micro-aspiration detection rates.

The strength of this study is robust symptom recording and the use of the validated RASP study to confirm reflux micro-aspiration and pharyngeal contamination.

\section{Conclusion}

The results of this study demonstrated that patients with reflux micro-aspiration most commonly present with a combination of a typical symptom of reflux such as heartburn and regurgitation and throat, voice or 
respiratory symptoms such as cough, voice change or throat clearing. Presence of an unexplained cough in settings of regurgitation or heartburn should prompt clinicians to interrogate for presence of reflux micro-aspiration for the risk of chronic pulmonary disease.

Provenance: Submitted article, peer reviewed.

Conflict of interest: None declared.

References

1 Morice AH, Dettmar PW. Reflux Aspiration and Lung Disease. Springer International Publishing, 2018.

2 Rey E, Elola-Olaso CM, Rodríguez-Artalejo F, et al. Prevalence of atypical symptoms and their association with typical symptoms of gastroesophageal reflux in Spain. Eur J Gastroenterol Hepatol 2006; 18: 969-975.

3 Vaezi MF. Atypical manifestations of gastroesophageal reflux disease. MedGenMed 2005; 7: 25.

4 Everett CF, Morice AH. Clinical history in gastroesophageal cough. Respir Med 2007; 101: 345-348.

5 Pomari C, Mauroner L, Paiano S, et al. Bronchial reacutization and gastroesophageal reflux: is there a potential clinical correlation? Ann Transl Med 2016; 4: 304.

6 Rosen R, Johnston N, Hart K, et al. The presence of pepsin in the lung and its relationship to pathologic gastro-esophageal reflux. Neurogastroenterol Motil 2012; 24: 129-e85.

7 Belafsky PC, Postma GN, Koufman JA. The validity and reliability of the reflux finding score (RFS). Laryngoscope 2001; 111: 1313-1317.

8 Dauer E, Thompson D, Zinsmeister AR, et al. Supraesophageal reflux: validation of a symptom questionnaire. Otolaryngol Head Neck Surg 2006; 134: 73-80.

9 Morice AH. On chronic cough diagnosis, classification, and treatment. Lung 2021; 199: 433-434.

10 Khoma O, Burton L, Falk MG, et al. Predictors of reflux aspiration and laryngo-pharyngeal reflux. Esophagus 2020; 17: 355-362.

11 Burton L, Falk GL, Parsons S, et al. Benchmarking of a simple scintigraphic test for gastro-oesophageal reflux disease that assesses oesophageal disease and its pulmonary complications. Mol Imaging Radionucl Ther 2018; 27: 113-120.

12 Morice AH. Airway reflux as a cause of respiratory disease. Breathe 2013; 9: 256-266.

13 Pearson JP, Parikh S, Orlando RC, et al. Review article: reflux and its consequences - the laryngeal, pulmonary and oesophageal manifestations. Conference held in conjunction with the 9th International Symposium on Human Pepsin (ISHP) Kingston-upon-Hull, UK, 21-23 April 2010. Aliment Pharmacol Ther 2011; 33: Suppl. 1, 1-71.

14 Crausaz FM, Favez G. Aspiration of solid food particles into lungs of patients with gastroesophageal reflux and chronic bronchial disease. Chest 1988; 93: 376-378.

15 Henderson RD, Woolfe CR. Aspiration and gastroesophageal reflux. Can J Surg 1978; 21: 352-354.

16 Özdemir P, Erdinç M, Vardar R, et al. The role of microaspiration in the pathogenesis of gastroesophageal reflux-related chronic cough. J Neurogastroenterol Motil 2017; 23: 41-48.

17 Lee JS, Collard HR, Raghu G, et al. Does chronic microaspiration cause idiopathic pulmonary fibrosis? Am J Med 2010; 123: 304-311.

18 Blondeau K, Mertens V, Vanaudenaerde BA, et al. Gastro-oesophageal reflux and gastric aspiration in lung transplant patients with or without chronic rejection. Eur Respir J 2008; 31: 707-713.

19 D'Ovidio F, Singer LG, Hadjiliadis D, et al. Prevalence of gastroesophageal reflux in end-stage lung disease candidates for lung transplant. Ann Thorac Surg 2005; 80: 1254-1260.

20 Mays EE, Dubois JJ, Hamilton GB. Pulmonary fibrosis associated with tracheobronchial aspiration. A study of the frequency of hiatal hernia and gastroesophageal reflux in interstitial pulmonary fibrosis of obscure etiology. Chest 1976; 69: 512-515.

21 Ravelli AM, Panarotto MB, Verdoni L, et al. Pulmonary aspiration shown by scintigraphy in gastroesophageal reflux-related respiratory disease. Chest 2006; 130: 1520-1526.

22 Lee AL, Goldstein RS. Gastroesophageal reflux disease in COPD: links and risks. Int J Chron Obstruct Pulmon Dis 2015; 10: 1935-1949.

23 Kantar A, Bernardini R, Paravati F, et al. Chronic cough in preschool children. Early Hum Dev 2013; 89: Suppl. 3, S19-S24.

24 Raghu G, Meyer KC. Silent gastro-oesophageal reflux and microaspiration in IPF: mounting evidence for anti-reflux therapy? Eur Respir J 2012; 39: 242-245.

25 Gaude GS. Pulmonary manifestations of gastroesophageal reflux disease. Ann Thorac Med 2009; 4: 115-123.

26 Posserud I, Ersryd A, Simrén M. Functional findings in irritable bowel syndrome. World J Gastroenterol 2006; 12: $2830-2838$.

27 Simpson SB. Refractory Gastro-oesophageal Reflux Disease and Laryngopharyngeal Reflux - Use the Bottom up Approach. Arch Gastroenterol Res 2020; 1: 95-104. 
28 Giuliano C, Wilhelm SM, Kale-Pradhan PB. Are proton pump inhibitors associated with the development of community-acquired pneumonia? A meta-analysis. Expert Rev Clin Pharmacol 2012; 5: 337-344.

29 Lambert AA, Lam JO, Paik JJ, et al. Risk of community-acquired pneumonia with outpatient proton-pump inhibitor therapy: a systematic review and meta-analysis. PLOS ONE 2015; 10: e0128004.

30 Decalmer S, Stovold R, Houghton LA, et al. Chronic cough: relationship between microaspiration, gastroesophageal reflux, and cough frequency. Chest 2012; 142: 958-964.

31 Kahrilas PJ, Smith JA, Dicpinigaitis PV. A causal relationship between cough and gastroesophageal reflux disease (GERD) has been established: a pro/con debate. Lung 2014; 192: 39-46. 